ARTICLE

\title{
Whole genome sequencing of skull-base chordoma reveals genomic alterations associated with recurrence and chordoma-specific survival
}

Jiwei Bai ${ }^{1,2,3,9}$, Jianxin Shi ${ }^{4,9}$, Chuzhong Li (10) 1,2,3,5,9, Shuai Wang ${ }^{1,9}$, Tongwu Zhang (1) ${ }^{4}$, Xing Hua ${ }^{4}$, Bin Zhu (D) ${ }^{4}$, Hela Koka4 ${ }^{4}$ Ho-Hsiang Wu (1) 4, Lei Song 4,6, Difei Wang ${ }^{4,6}$, Mingyi Wang (D) 4,6, Weiyin Zhou ${ }^{4,6}$, Bari J. Ballew ${ }^{4,6}$, Bin Zhu ${ }^{4,6}$, Belynda Hicks (10 4,6, Lisa Mirabello4, Dilys M. Parry ${ }^{4}$, Yixuan Zhai ${ }^{1,7}$, Mingxuan Li ${ }^{1}$, Jiang Du1,3,5, Junmei Wang1,3,5, Shuheng Zhang 1,8, Qian Liu1, Peng Zhao 2,3, Songbai Gui 2,3, Alisa M. Goldstein (1) 4, Yazhuo Zhang (1) ${ }^{1,2,3,5 \bowtie}$ \& Xiaohong R. Yang ${ }^{4}$

Chordoma is a rare bone tumor with an unknown etiology and high recurrence rate. Here we conduct whole genome sequencing of 80 skull-base chordomas and identify PBRM1, a SWI/ SNF (SWItch/Sucrose Non-Fermentable) complex subunit gene, as a significantly mutated driver gene. Genomic alterations in PBRM1 (12.5\%) and homozygous deletions of the CDKN2A/2B locus are the most prevalent events. The combination of PBRM1 alterations and the chromosome 22q deletion, which involves another SWI/SNF gene (SMARCB1), shows strong associations with poor chordoma-specific survival (Hazard ratio $[H R]=10.55,95 \%$ confidence interval $[\mathrm{Cl}]=2.81-39.64, \mathrm{p}=0.001)$ and recurrence-free survival $(\mathrm{HR}=4.30$, $\left.95 \% \mathrm{Cl}=2.34-7.91, \mathrm{p}=2.77 \times 10^{-6}\right)$. Despite the low mutation rate, extensive somatic copy number alterations frequently occur, most of which are clonal and showed highly concordant profiles between paired primary and recurrence/metastasis samples, indicating their importance in chordoma initiation. In this work, our findings provide important biological and clinical insights into skull-base chordoma.

\footnotetext{
${ }^{1}$ Beijing Neurosurgical Institute, Capital Medical University, Beijing, China. ${ }^{2}$ Department of Neurosurgery, Beijing Tiantan Hospital, Capital Medical University, Beijing, China. ${ }^{3}$ China National Clinical Research Center for Neurological Diseases, Beijing, China. ${ }^{4}$ Division of Cancer Epidemiology and Genetics, National Cancer Institute, NIH, DHHS, Bethesda, MD, USA. ${ }^{5}$ Brain Tumor Center, Beijing Institute for Brain Disorders, Beijing, China. ${ }^{6}$ Cancer Genomics Research Laboratory, Leidos Biomedical Research, Frederick National Laboratory for Cancer Research, Frederick, MD, USA. ${ }^{7}$ Department of Neurosurgery, The First Affiliated Hospital of Zhengzhou University, Zhengzhou, China. ${ }^{8}$ Department of Neurosurgery, Anshan Central Hospital, Anshan, China. ${ }^{9}$ These authors contributed equally: Jiwei Bai, Jianxin Shi, Chuzhong Li, Shuai Wang. ${ }^{凶}$ email: zhangyazhuo@ccmu.edu.cn
} 
C hordoma is a rare bone tumor, which is believed to originate from notochordal remnants ${ }^{1}$ and occurs in the axial skeleton of cranial, vertebral, and sacral sites ${ }^{2}$. Based on the United States Surveillance Epidemiology and End Results data, the incidence of chordoma varies by gender and race $^{3}$, however, little is known about the etiologic factors that predispose to it. Germline duplication of the TBXT gene, which encodes brachyury, a transcription factor that plays an important role in embryonic development, was identified as a major susceptibility mechanism in familial chordoma ${ }^{4}$. A common genetic polymorphism in TBXT was subsequently associated with an increased risk for both familial and sporadic chordoma ${ }^{5,6}$.

Chordomas are considered slow growing; however, the recurrence rate is high, especially among skull-base chordoma patients, largely due to incomplete tumor resection. The clinical progression of skull-base chordoma is highly variable ${ }^{7}$, and there are no validated clinical or molecular prognostic panels available. Treatment for a skull-base tumor usually involves surgery with or without adjuvant radiation therapy (RT). Chemotherapy or other systemic therapies are not effective for treating chordoma. Although several potentially druggable molecular targets have been identified and some are being evaluated in clinical trials ${ }^{8}$, treatment options for chordoma patients, particularly those with advanced disease, are still limited. A better understanding of the

\section{Table 1 Characteristics of chordoma patients included in the} whole-genome sequencing analysis $(n=80)^{\text {a. }}$.

\section{N (\%)}

Age (year) ${ }^{\mathrm{b}}$

$\leq 20$ $44.7(7-79)$

$20-40$

$10(12.5)$

$40-50$

$50+$

$21(26.2)$

$14(17.5)$

$35(43.8)$

Sex

$\mathrm{F}$

M

$30(37.5)$

$50(62.5)$

Histological type

Classic

$64(80.0)$

Chondroid

Dedifferentiated

$14(17.5)$

$2(2.5)$

Surgery type

Endoscopic endonasal $\quad 78$ (97.5)

Open craniotomy

Gross resection rate

Complete

Near complete

Subtotal or partial

Tumor volume $\left(\mathrm{cm}^{3}\right)^{\mathrm{b}}$

Presurgery RTC treatment

No

Yes

$2(2.5)$

$16(20.0)$

$36(45.0)$

$28(35.0)$

$35.0(4.2-147.5)$

$68(85.0)$

$12(15.0)$

Post-surgery $\mathrm{RT}^{\mathrm{c}}$ treatment

No

$38(47.5)$

Yes

$42(52.5)$

Recurrence status

No

$21(26.3)$

$59(73.7)$

Yes

$21(1-128)$

RFS' $^{d}$ (month) ${ }^{b}$

Death status

$\begin{array}{ll}\text { No } & 63(78.8)\end{array}$

Yes $17(21.2)$

Survival (month) ${ }^{\mathrm{b}} \quad 50(10-157)$

aNone of these patients received any chemo, immune, or targeted therapies.

bCharacteristic in its continuous form is expressed as mean (range).

CRadiation therapy.

Recurrence-free survival. molecular processes in chordoma is critically needed to develop prognostic prediction tools and to discover druggable targets.

Genomic profiling studies of chordoma are limited. The largest sequencing analysis so far included 104 sacral chordoma patients, but whole-genome sequencing (WGS) was conducted on only 11 tumors 9 . Results from this study suggested that amplifications of TBXT (encoding brachyury), homozygous deletion of CDKN2A, and mutations in SWI/SNF (SWItch/Sucrose Non-Fermentable) complex genes (PBRM1, SETD2, ARID1A) and the PI3K signaling pathway were the most frequent genomic events in sacral chordoma. Limited by the small number of WGS and WES samples analyzed, formal testing for driver gene mutations, structural variants, and mutation signatures was not conducted. It also remains unclear whether skull-base chordoma, which is associated with a much earlier age onset (47.4 years) compared to sacral chordoma (62.7 years $)^{3}$, is driven by similar genomic alterations. Furthermore, the clinical relevance of these genomic events is largely unknown.

Here in this chordoma WGS study, we provide a detailed genomic landscape of skull-base chordoma, which revealed potential driver events, mutational signatures, and outcomerelated genomic features. Our findings suggest that the combination of SWI/SNF alterations and $22 \mathrm{q}$ deletion show a strong association with clinical outcomes, demonstrating the potential of designing a multi-marker panel in prognostic prediction.

\section{Results}

Patient characteristics. The primary analysis included 80 patients with skull-base chordoma who were diagnosed and treated at Beijing Tiantan Hospital, China. The detailed clinical characteristics of these patients are shown in Table 1. In brief, the mean age at initial diagnosis of chordoma among these patients was 44.7 years (range: 7-79); 62.5\% were males, the majority (80\%) had conventional/classical chordoma, and 12 received RT prior to surgery. After an average follow-up period of 50 months, there were 59 recurrences and 17 deaths, all died of chordoma (Table 1).

We performed WGS on 91 surgically resected chordomas from 80 patients (including 11 paired primary and recurrent tumor samples) and their matched germline DNA from whole blood, with the average sequencing depth of $41 \mathrm{x}$ for blood and $76 \mathrm{x}$ for tumor samples (Supplementary Fig. 1). The average tumor purity estimated from copy number $(\mathrm{CN})$ alterations was $57 \%$.

In a separate analysis, we also analyzed a patient with metastatic chordoma, who was diagnosed with chordoma at 17 years old. During post-surgery RT, her tumor was found to have recurred in the skull base and to have metastasized into the lymph node, thorax, and liver. We conducted WGS on the chordoma recurrence sample, lymph node metastasis (LM), thoracic metastasis (TM), and their matched germline blood samples.

Germline susceptibility. TBXT is the only chordoma susceptibility gene identified to date. Germline TBXT gene duplication was reported in a subset of chordoma families ${ }^{4}$ but was rare in sporadic chordoma patients ${ }^{6}$. Using matched germline WGS data, we found germline TBXT gene duplication in a single patient (P22, Supplementary Fig. 2), who was 47 years old at diagnosis and had local recurrence. We then evaluated rare exonic variants in known cancer predisposition genes ${ }^{10}$ or previously reported chordoma-related genes (including potential germline susceptibility and somatically mutated genes, Supplementary Table 1) and found two variants, each in a single patient, that were classified as pathogenic (ERCC5, c.697 C>T, p. Gln233*) or likely pathogenic (LP) (BLM, c.3564delC, p. Phe1189fs) (see classification criteria in "Methods"). Both 
patients were heterozygous carriers, and the evaluation of somatic mutation and $\mathrm{CN}$ data did not find mutations or losses of the other allele in either primary tumor or recurrence samples in these two patients. In summary, our data did not support a strong germline contribution from known cancer predisposition genes or chordoma genes in this patient cohort.

A common genetic variant in the TBXT gene (rs2305089) was previously associated with a sixfold increase in risk of developing chordoma in a European population ${ }^{5}$, however, this variant was not significantly associated with chordoma risk in a Chinese study of skull-base chordoma ${ }^{11}$. In our study, the frequency of the variant allele $(A)$ among chordoma patients is $33.7 \%$, which is similar to what was reported in the Chinese study by $\mathrm{Wu}$ et al. ${ }^{11}$ and is much lower than those reported in European chordoma patients $(>80 \%)$. The frequency of the variant allele observed among chordoma patients in our study is also similar to those reported in the general population among East Asians, further implicating that this variant is not associated with skull-base chordoma among Chinese.

Somatic genomic landscape. Among 80 distinct tumors in the primary analysis, most had low tumor mutational burden (TMB, median $=0.53$ mutations $/ \mathrm{Mb}$, range $=0.05-7.68$, per tumor $)$. TMB was lower compared to most cancer types sequenced in The Cancer Genome Atlas (TCGA) (Supplementary Fig. 3). Numbers of single nucleotide variations (SNVs), insertion and deletions (indels), and structural variants (SVs) in these patients are shown in Fig. 1. Patients who received presurgery RT $(n=12)$ tended to have higher TMB $($ median $=0.75$ mutations $/ \mathrm{Mb}$, range $=0.055-1.56$ ) compared with patients without presurgery treatment $(n=68$, median $=0.49$ mutations $/ \mathrm{Mb}$, range $=0.05-7.68)$, however, the difference was not statistically significant $(p=0.42)$. The average number of SVs per tumor was 38. The most prevalent SV events included deletions (13\%), deletions with insertions (16\%), and chromosomal translocations (13\% for inter-chromosome and $10 \%$ for intra-chromosome translocations) (Fig. 1).

Mutational signatures. SigProfiler (https:/github.com/ AlexandrovLab) ${ }^{12}$ was used to identify single-base substitution (SBS), double-base substitution (DBS), and small indel (ID) mutational signatures. We identified nine de novo SBS mutation patterns in 80 primary tumors that were highly correlated with combinations of existing COSMIC signatures (cosine similarities ranging from 0.84 to 0.99; Supplementary Fig. 2A). No novel SBS patterns were identified in this analysis.

Since the observed de novo signatures largely overlapped with known COSMIC signatures, we therefore focused on the contributions of the known signatures to the mutational landscape of our chordoma cohort. The predominant SBS signature is SBS5 (mean fraction $=67.9 \%$, range $=0-94.9 \%$ ), which is a common clock-like signature in many cancer types. Other prevalent SBS signatures included SBS8 (9.9\%), SBS1 (10.0\%), and SBS40 (5.4\%) (Fig. 1 and Supplementary Table 2A). SBS1 is associated with the deamination of 5-methylcytosine, reflecting age-related accumulation. SBS8, which was recently associated with nucleotide excision repair deficiency in breast tumors $^{13}$, was associated with higher TMB (Spearman correlation, $p=0.017)$. The average fractions of APOBEC signatures (SBS2: $1.1 \%$ and SBS13: $1.2 \%$ ), which were low in this patient cohort (Fig. 1 and Supplementary Table 2A), were associated with higher number of SVs $(p=0.002)$. Two tumors (P21 and P73), in which TMB was among the highest, showed high contributions of SBS44, which is a signature associated with defective DNA mismatch repair (MSI). One of these tumors (P21) carried nonsynonymous mutations in MLH1 and TP53, showing a characteristic of high MSI. Interestingly, both tumors had alterations in SETD2 (one missense mutation predicted to be damaging by PolyPhen and Sift and one SV).

We also identified five de novo indel signature patterns, four of which reflected combinations of COSMIC ID1-6, 8, and 9 (cosine similarities ranging from 0.93 to 1.00; Supplementary Fig. 4B). The remaining one (signature A), which is composed predominantly of $2 \mathrm{bp}$ insertions (mainly AT and TA) at long $(\geq 5)$ repeats, was not mapped to any COSMIC ID signatures (Fig. 1). This signature was prevalent in chordoma tumors, which was present in 55 of 80 tumors (Supplementary Table 2B). The number of mutations per tumor sample attributed to this signature was not associated with any patient characteristics or genomic features such as age, sex, presurgery or post-surgery RT, TMB, or any SBS/DBS signatures.

When conducting the mutational signature analysis separately for clonal and subclonal mutations classified by PyClone ${ }^{14}$, we found that fractions of clonal and subclonal signatures were highly correlated. No significant differences were found between clonal and subclonal analyses (Supplementary Fig. 5).

When comparing the mutational profiles and signatures of chordoma against those of 9450 tumor samples comprised of different cancer types using MutaGene (https://www.ncbi.nlm. nih.gov/research/mutagene/ $/)^{15}$, we found that the mutational profiles of chordoma were most similar to those of kidney cancers (clear cell and papillary cell), chronic lymphocytic leukemia, and bone cancer (Supplementary Table 3).

Recurrently mutated genes. When restricting to nonsynonymous mutations (see "Methods" for definition), we found that PBRM1 (6.25\%), B2M (3.75\%), and MAP3K4 (3.75\%) were the most frequently mutated known cancer driver genes ${ }^{16}$ in this patient cohort. Driver gene analysis combining SNVs and indels using dNdScv (v0.1.0, https://github.com/im3sanger/dndscv/ releases/tag/0.1.0 $)^{17}$ identified only one significantly mutated gene, $P B R M 1$, at false discovery rate (FDR) $q<0.1(q=0.0001)$. Five different $P B R M 1$ mutations were found, each in a different patient (Fig. 2). We also found two mutations in LYST (each in a single tumor), a gene that encodes a protein regulating intracellular protein trafficking in endosomes and was previously suggested as a potential chordoma driver gene ${ }^{9}$. One of the two LYST mutation carriers also harbored a germline TBXT duplication. TP53 mutations, which are common across different cancer types, were only seen in one chordoma patient. Mutations in other known cancer driver genes were detected in 17 additional tumors, each only occurred in a single sample (Fig. 2), indicating substantial heterogeneity in the driver mutation landscape.

For mutations in noncoding regions, we focused on mutations in the promoter region of known cancer driver genes, and we did not detect mutations in any of these genes including TERT in our patient cohort.

Somatic copy number alterations (SCNAs) and structural variants (SVs). Using FACETS ${ }^{18}$, we identified arm/chromosome-level SCNAs in the majority $(77.5 \%)$ of primary chordoma samples. Consistent with previous reports ${ }^{19,20}$, we observed frequent arm-level SCNA events (Fig. 3a). Among them, 17 events were found to be significant $(q<0.1)$ by GISTIC (https://software. broadinstitute.org/cancer/cga/gistic) analysis ${ }^{21}$, including gains of chromosomes $1 \mathrm{q}, 7 \mathrm{p}$, and $7 \mathrm{q}$, and deletions of $1 \mathrm{p}, 3,4,9,10,13 \mathrm{q}$, $14 \mathrm{q}, 18$, and 22q. Clustering analysis revealed five distinct groups of patients based on SCNA events. Group $1(n=16)$ and Group 2 $(n=25)$ demonstrated extensive SCNAs, with Group 2 lacking chromosome gains and Group 1 lacking deletions of 4, 9, and 14 . Group $3(n=13)$ had scattered SCNAs, most of which are 

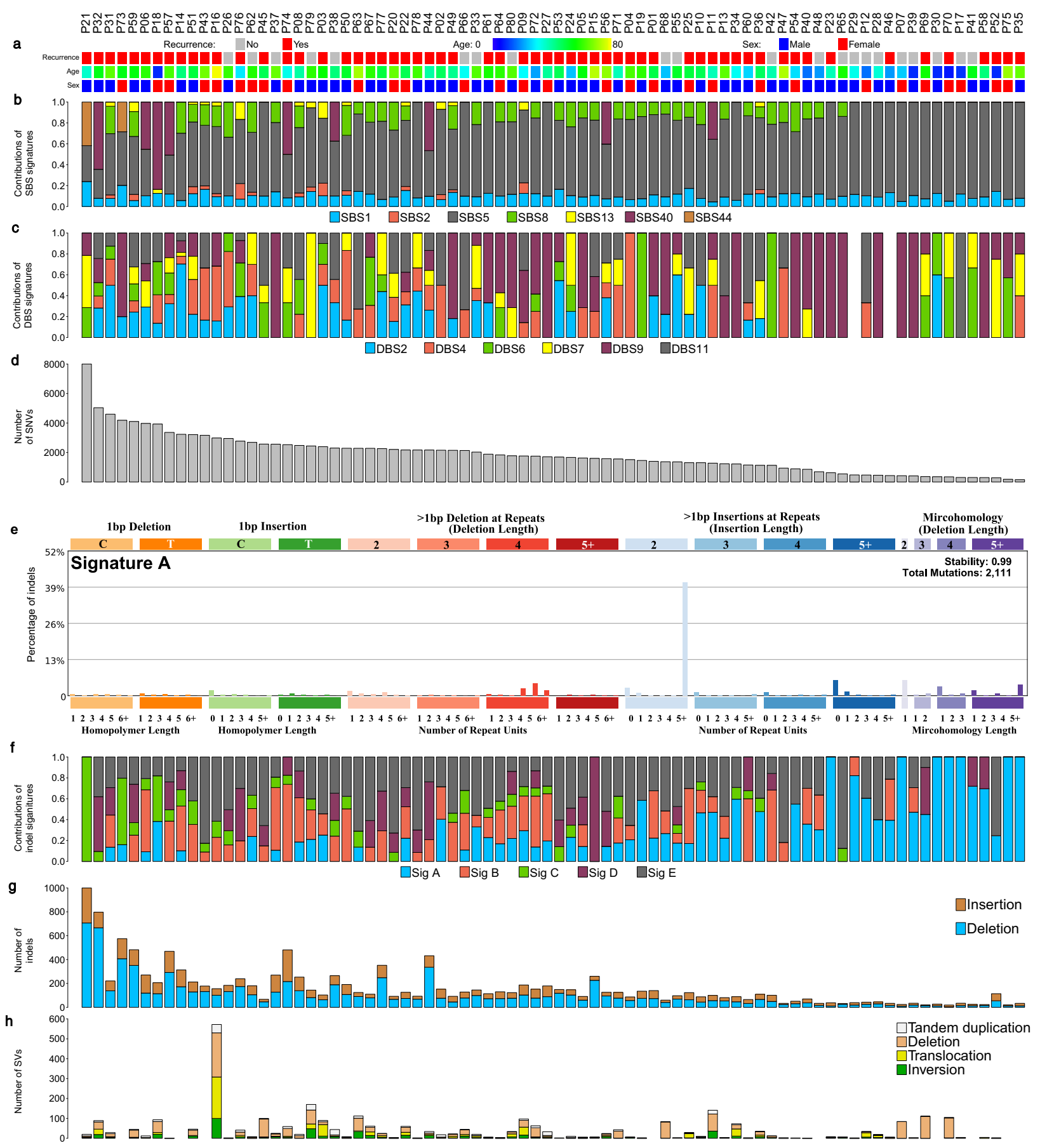

Fig. 1 Mutational landscape of skull-base chordoma $(\boldsymbol{n}=\mathbf{8 0})$. a The recurrence status (no: gray; yes: red), age (from younger to older: blue to yellow), and sex (female: red; male: blue); $\mathbf{b}$ the proportions of somatic single-base substitutions (SBS) found in each chordoma patient that can be attributed to COSMIC SBS signatures; $\mathbf{c}$ the proportions of somatic double-base substitutions (DBS) found in each chordoma patient that can be attributed to COSMIC DBS signatures; $\mathbf{d}$ number of single nucleotide variants (SNVs) identified in each chordoma sample; $\mathbf{e}$ the de novo signature $\mathrm{A}$, which is not mapped to any of COSMIC indel signatures; $\mathbf{f}$ the proportions of de novo indels found in each chordoma patient; $\mathbf{g}$ number of indels found in each chordoma sample; h number of structural variants (SVs) found in each chordoma sample. See also Supplementary Figs. 4 and 5 and Tables 2 and 3.

deletions. Group $4(n=19)$ had no or few SCNAs. Group $5(n=$ 7) was characterized by the enrichment for extensive chromosome gains throughout the genome (Fig. 3a), a characteristic consistent with whole-genome doubling (WGD), defined by $50 \%$ of autosomal genome having an MCN (the more frequent allele in a given segment) greater than or equal to two (Supplementary
Table 4$)^{22}$. The number of nonsynonymous SNVs and SVs did not vary significantly among the five SCNA groups.

GISTIC analysis of focal SCNA regions identified 6 significant $(q<0.1)$ amplifications and 12 significant deletion regions (Supplementary Fig. 6A). The most significant deletion region was 9 p21.3 $\left(p=5.87 \times 10^{-13}\right)$, which contains the known tumor 


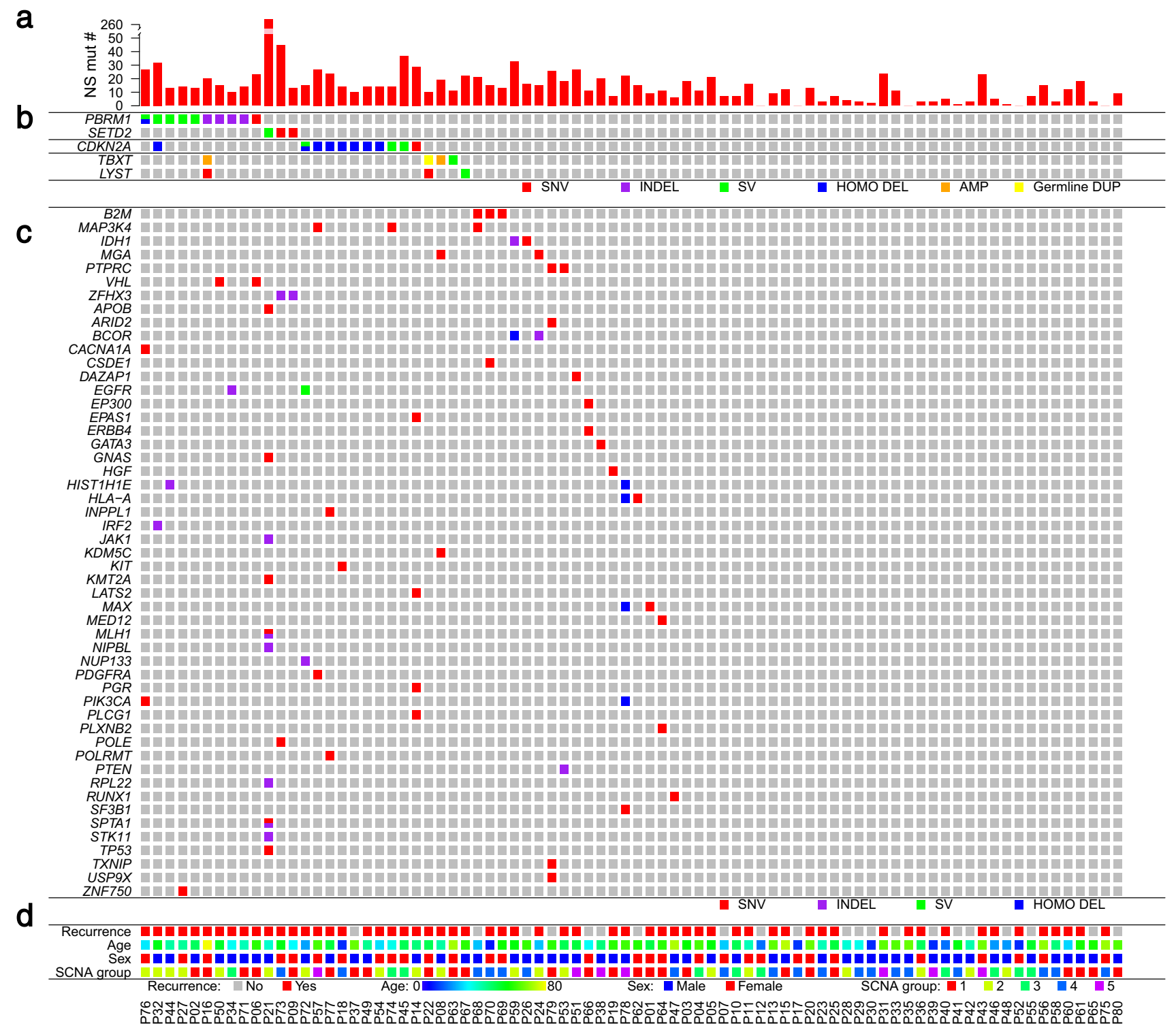

Fig. 2 Genomic driver landscape of skull-base chordoma. a The number of nonsynonymous (NS) mutations per tumor. $\mathbf{b}$ The potential chordoma driver genes. c The middle gene panel reports NS mutations in known cancer driver genes that were detected in this study. SNV single nucleotide variant, SV structural variant, HOMO DEL homozygous deletion, AMP amplification, Germline DUP germline duplication. $\mathbf{d}$ The recurrence status (no: gray; yes: red), age (from younger to older: blue to yellow), sex (female: red; male: blue), and SCNA group (see Fig. 3). See also Supplementary Fig. 2.

suppressor gene $C D K N 2 A$, while the peak region of the $3 \mathrm{p} 21.1$ deletion $(p=0.062)$ contained PBRM1 and SETD2, both are chromatin remodeling genes. In a subset of patients with RNA sequencing (RNA-Seq) data available $(n=27)$, we found that patients with $3 \mathrm{p} 21.1$ deletion had decreased expression of PBRM1 $(p=0.025)$ and SETD2 $(p=0.033)$ (Supplementary Fig. 6B). In contrast to reported frequent somatic duplications of TBXT $(>20 \%)$ as a driver event in sacral chordoma ${ }^{9}$, focal TBXT duplications were only seen in two tumors (P08 and P16) in our patient cohort, which did not reach statistical significance.

We used the Meerkat algorithm ${ }^{23}$ to identify SVs and used ShatterSeek (v0.4) to detect chromothripsis (clusters of SVs) ${ }^{24}$. Similar to what was previously reported in glioblastoma ${ }^{23}$, we found complex genomic rearrangement events involving the $C D K N 2 A / 2 B$ locus (Fig. 3b). Thirty-one (40\%) tumors had armlevel $9 \mathrm{p}$ deletion, 9 of which also had focal deletions or complex rearrangements of the $9 \mathrm{p} 21.3$ region resulting in the homozygous loss of the CDKN2A/2B locus (Fig. $3 \mathrm{~b}$ ).
Twelve high-confidence chromothripsis events were detected in seven tumors (Supplementary Table 5), with two events (in P16 and P25) involving chromosome $3 \mathrm{p}$, including the PBRM1 gene region (Fig. 3c). We also observed extensive chromothripsis of the chromosome $6 \mathrm{q}$ region encompassing the TBXT gene in one tumor (P63, Fig. 3d).

Summary of driver landscape of skull-base chordoma. To characterize the potential driver events for skull-base chordoma, we combined five patients with PBRM1 mutations and five patients with SVs involving PBRM1 (Fig. 4), which were validated by targeted sequencing. Together with SETD2 alterations in three patients, alterations in these two chromatin remodeling genes accounted for $16 \%$ (13 of 80 ) of the chordoma tumors we sequenced (Fig. 2). Genomic alterations involving the $C D K N 2 A / B$ loci were the next prevalent event $(C D K N 2 A /+, 13.8 \%$ patients, nine with 9p21.3 homozygous deletion, one with SVs involving 
a

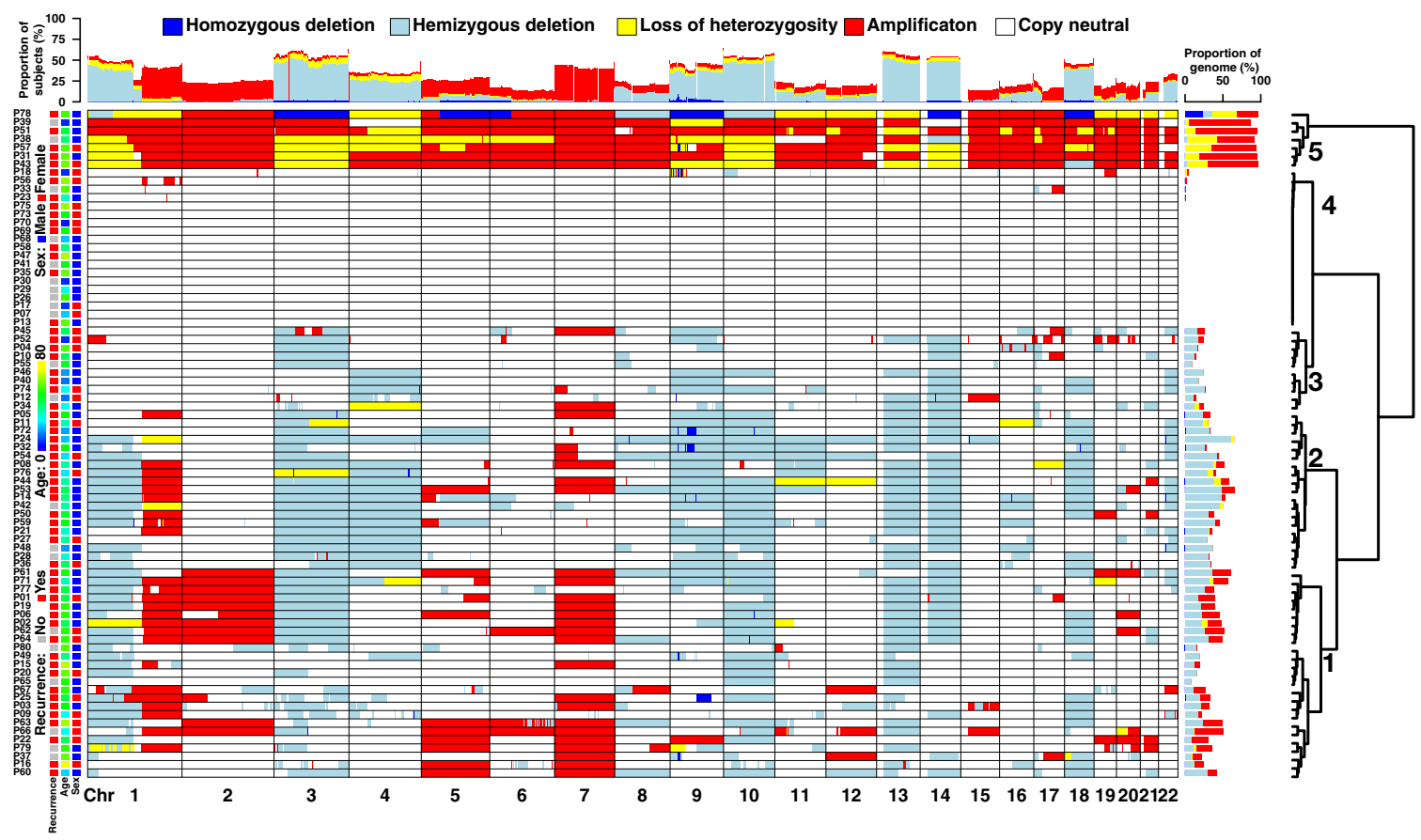

b

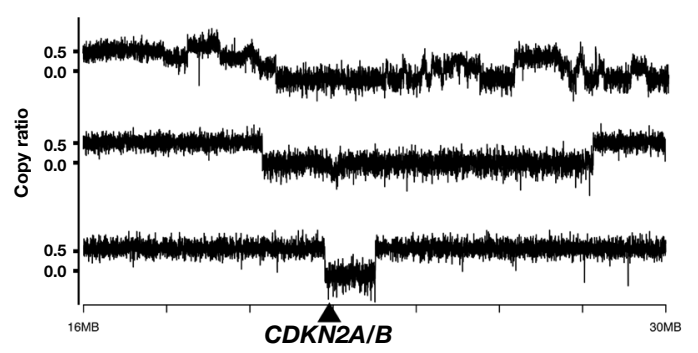

C

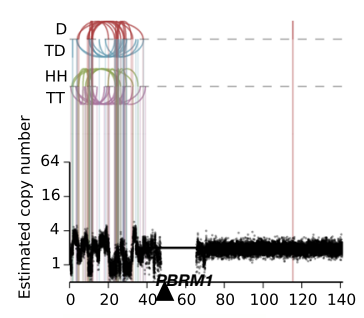

d

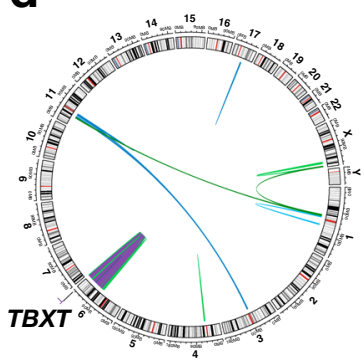

Fig. 3 Somatic copy number alterations (SCNAs) and structural variants (SVs) in skull-base chordoma patients. a Genome-wide SCNAs. Patients were clustered into five groups. Group 1: extensive SCNAs, lacking deletions of 4, 9, and 14; Group 2: extensive SCNAs, lacking amplifications; Group 3: scattered SCNAs, most of which are deletions; Group 4: no or few SCNAs; and Group 5: extensive amplifications demonstrating characteristic consistent with wholegenome doubling. b Representative examples showing complex rearrangements at chromosome 9p. Plots show copy ratios (tumor vs. matched normal) of the chromosome 9p21 region. All three samples show an arm-level deletion coupled with complex rearrangements (top) and focal deletions of the 9p21.3 region (middle) or the CDKN2A/2B locus (bottom), resulting in the homozygous loss of CDKN2A/2B. c An example of complex rearrangements at chromosome 3p. D deletion, TD tandem duplication, HH head-to-head inverted, TT tail-to-tail inverted. $\mathbf{d}$ A chordoma sample showing chromothripsis of the $6 \mathrm{q}$ region, where the TBXT gene is located. See also Supplementary Tables 4 and 5.

the $C D K N 2 A / 2 B$ loci, and one with $C D K N 2 A$ mutation), which occurred mutually exclusively with PBRM1 and SETD2 alterations (Fig. 2). Genomic alterations disrupting the TBXT gene $(T B X+)$ were only observed in four patients, including a patient with germline TBXT duplication, two tumors with somatic focal TBXT duplication, and one tumor with $6 \mathrm{q}$ chromothripsis. Disruptions of LYST (LYS+) were seen in three tumors; one of them also had a PBRM1 mutation and another had TBXT germline duplication.

In summary, we identified candidate driver events (PBRM1+, SETD2+, CDKN2A/B+, TBXT/LYST+) in 33.75\% (27 out of 80 ) of the skull-base chordoma patients we sequenced. The remaining tumors might be caused by nonsynonymous mutations or SCNAs/SVs in known cancer driver genes (observed in $8 \%$ patients, Fig. 2) or other driver genes/mechanisms for which statistical power was too low to detect in this study. In particular, some of these tumors showed extensive chromosomal SCNAs that are consistent with chromosomal aneuploidy (Supplementary Fig. 7), which may drive chordoma development, similar to what has been previously reported in chromophobe renal cell carcinomas and pancreatic neuroendocrine tumors that also showed high fractions of tumors without known drivers ${ }^{25}$.

$P B R M 1+$ tumors had higher total mutation (Wilcoxon rank sum, $p=0.04)$ and nonsynonymous mutation burden $(p=0.07)$, were more likely to be in SCNA group 1 (reference: SCNA Groups 3 and $4 ; p=0.02$ ), had higher fractions of $A P O B E C$ mutational signatures $(p=0.09)$, and higher number of SVs, particularly tandem duplications $(p<0.0001)$ and interchromosome translocations $(p=0.03)$, as compared to tumors without PBRM1 alterations (PBRM1-). PBRM1+ tumors had reduced expression of PBRM1 $(p=0.16)$ compared to PBRM1tumors, although the difference was not statistically significant 


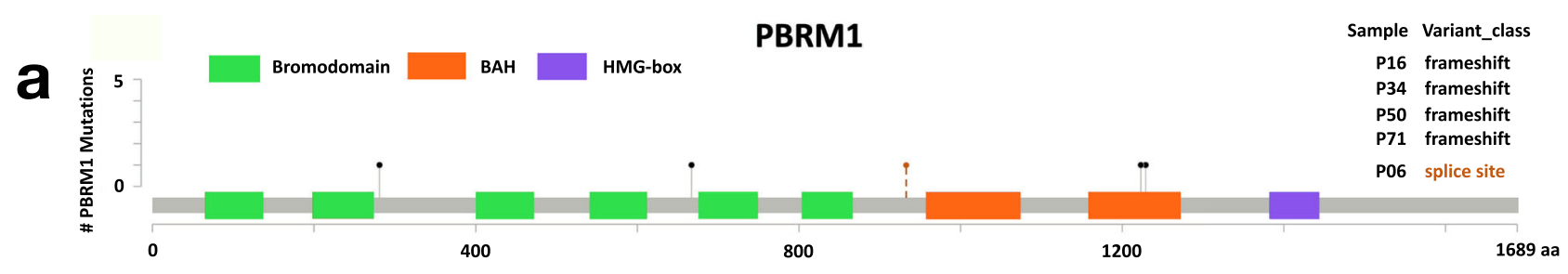

b an
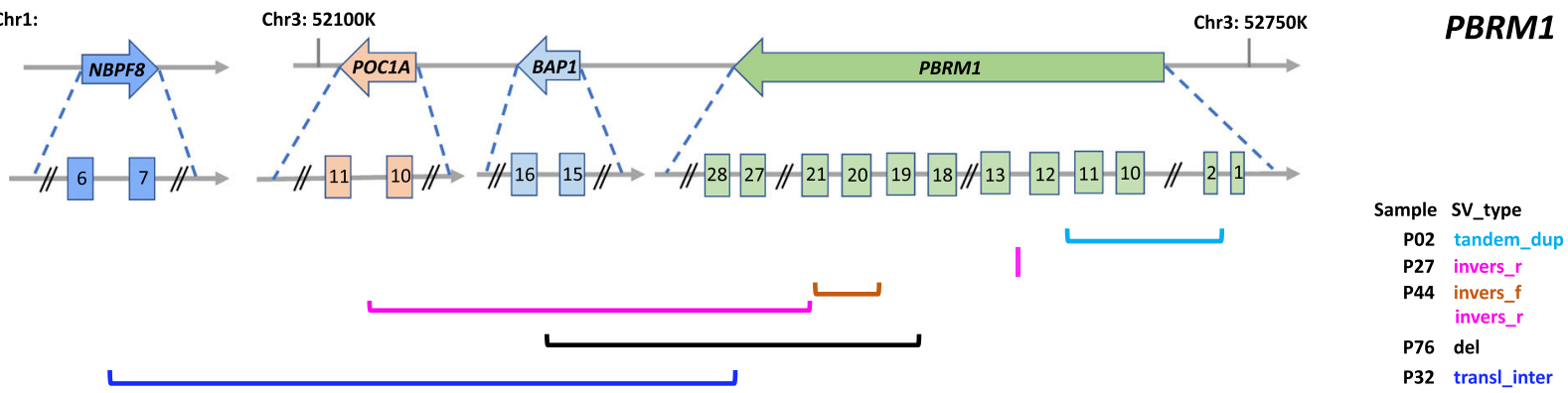

Fig. 4 Summary of PBRM1 alterations identified in ten skull-base chordoma patients. a Lollipop plots depict nonsynonymous mutations found in $P B R M 1$ (five patients), showing identified mutations relative to a schematic representation of the gene. Each lollipop denotes a unique mutation and the length of the line reflects the number of samples with the mutation. The colored boxes are functional domains of the protein. The splicing mutation, which is located in a noncoding region, is shown with a broken line. b Structural variants (SVs) found in PBRM1 (five patients) detected by Meerkat. Different SV types are indicated by different color schemes.

(Supplementary Fig. 8A). Similarly, CDKN2A/B+ tumors had lower CDKN2A expression level $(p=0.003$, Supplementary Fig. $8 \mathrm{~B}$ ) and they were also more likely to have tumors with higher nonsynonymous mutation burden $(p=0.002)$. Associations between mutation burden and alterations in PBRM1 $(p=$ $0.12)$ and $C D K N 2 A / 2 B(p=0.06)$ were similar but less significant (possibly due to smaller sample size) when restricting to patients without presurgery RT.

Somatic driver landscape in relation to patient characteristics. Among the 80 distinct patients with WGS data, 64 had conventional/classical, 14 had chondroid, and 2 had dedifferentiated chordoma. Mutational burden, total numbers of SVs, and driver genomic events (PBRM1 and $C D K N 2 A / B)$ did not vary significantly between conventional and chondroid chordoma tumors. Both patients with dedifferentiated chordoma had 9p21 homozygous deletions as well as high mutational burden and complex structural alterations. One of them was diagnosed with chordoma at age 9 years old and her tumor recurred 6 months after surgery. In contrast, the other nine pediatric chordoma patients (diagnosed $\leq 20$ years) appeared to have quiet genomes, characterized by low mutational burden and the absence of driver events (alterations in PBRM1, CDKN2A/B, TBXT genes), compared with adult chordoma patients.

Somatic driver landscape and clinical prognosis. As it is clinically important and challenging to identify chordoma patients with aggressive features, we sought to identify genomic alterations that were associated with patient outcomes (17 deaths and 59 recurrences). We first examined the two potential driver events, $P B R M 1$ and $C D K N 2 A / B$ status, in relation to chordoma-specific survival (CSS) and recurrence-free survival (RFS). After adjustment for age, sex, presurgery and post-surgery RT, PBRM1 alterations were significantly associated with worse CSS (hazard ratio $(\mathrm{HR})=4.79,95 \%$ confidence interval $(\mathrm{CI})=1.57-14.59$, $p=0.0058)$ and RFS (HR $=5.72,95 \% \mathrm{CI}=2.68-12.19, p=6.4 \times$ $10^{-6}$ ) (Fig. 5).
In contrast, $C D K N 2 A / B+$ status (9p21.3 homozygous deletion, SVs involving the $C D K N 2 A / 2 B$ loci, and $C D K N 2 A$ mutation) was not significantly associated with $\mathrm{CCS}(\mathrm{HR}=0.88,95 \% \mathrm{CI}=$ $0.20-3.92, p=0.86)$ or RFS (HR $=1.68,95 \% \mathrm{CI}=0.66-4.31, p=$ $0.28)$. However, when we looked at $9 \mathrm{p}$ and $9 \mathrm{q}$ arm-level deletions and focal SCNAs on chromosome 9, we found that arm-level 9q deletion and focal deletions of $9 \mathrm{p} 11.2,9 \mathrm{p} 21.3$, and $9 \mathrm{q} 21.11$ were significantly associated with RFS (Fig. 5 and Supplementary Table 6). Interestingly, the RFS association was stronger for 9q21.11 focal deletion $(\mathrm{HR}=3.63,95 \% \mathrm{CI}=1.97-6.69, p=$ $3.44 \times 10^{-5}$ ) as compared to deletions of the $9 \mathrm{p} 21.3$ region, where CDKN2A/2B is located $(\mathrm{HR}=2.65,95 \% \mathrm{CI}=1.42-4.93, p=$ $0.002)$. These findings suggest that the RFS association was not entirely driven by $C D K N 2 A / 2 B$ alterations.

Next, we tested the associations between 17 significant armlevel SCNA events and clinical outcomes. After multiple testing correction, we found that $22 \mathrm{q}$ deletion was significantly associated with CSS $(\mathrm{HR}=5.88,95 \% \mathrm{CI}=1.85-18.68$, nominal $p=0.0027$, Bonferroni corrected $p=0.046)$ and RFS ( $\mathrm{HR}=3.74,95 \% \mathrm{CI}=$ 1.89-7.38, nominal $p=0.00014$, Bonferroni corrected $p=0.0024$ ) (Supplementary Table 6). Chromosome 22q harbors an important SWI/SNF gene, SMARCB1/IN1, and the complete loss of SMARCB1 expression on immunohistochemistry due to homozygous SMARCB1 deletion has been used as a marker for a rare chordoma subtype, poorly differentiated chordoma ${ }^{8,26}$. Homozygous deletion of SMARCB1 was not seen in our patients. However, we found that RNA expression of SMARCB1 was significantly lower in tumors with arm-level chromosome 22q deletion compared to those without the deletion in a subset of patients with RNA-Seq data available ( $p=0.001$, Supplementary Fig. 8C).

When we combined the two significant events for CSS (PBRM1 and $22 \mathrm{q}$ deletion), the association for CSS became slightly stronger $(\mathrm{HR}=10.55,95 \% \mathrm{CI}=2.81-39.64, p=0.001)$. Similarly, the combination of PBRM1,9q21.11 deletion, and 22q deletion also improved the association for RFS ( $\mathrm{HR}=4.22,95 \%$ $\mathrm{CI}=2.34-7.62, p=1.77 \times 10^{-6}$; comparing harboring any event to no event). HRs for the associations between these genomic 


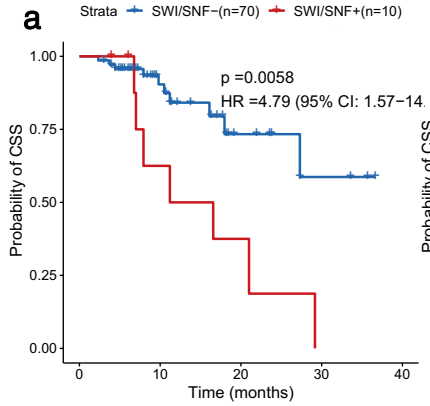

e Strata - SWI/SNF-(n=70) - SWI/SNF+(n=10)

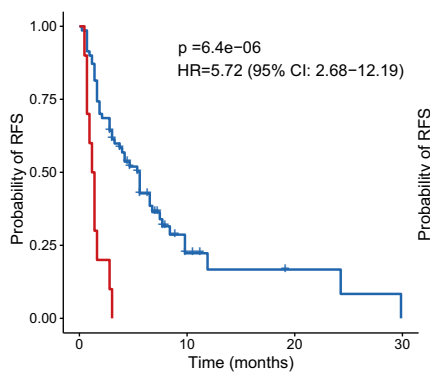

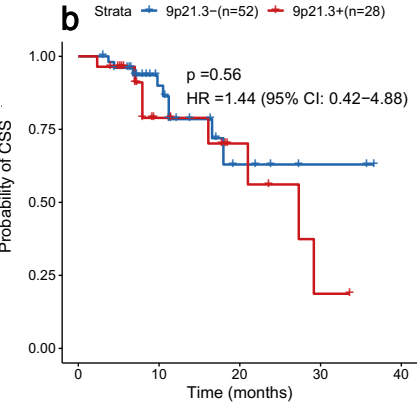
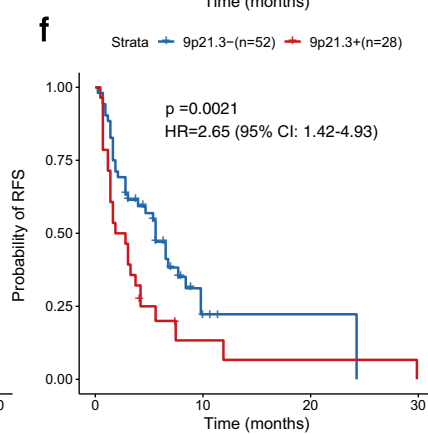

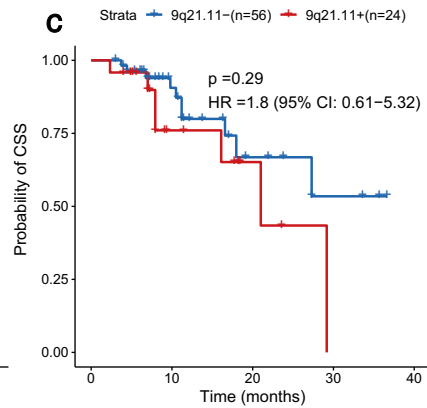

g Strata $+9 \mathrm{q} 21.11-(\mathrm{n}=56)+9 \mathrm{q} 21.11+(\mathrm{n}=24)$

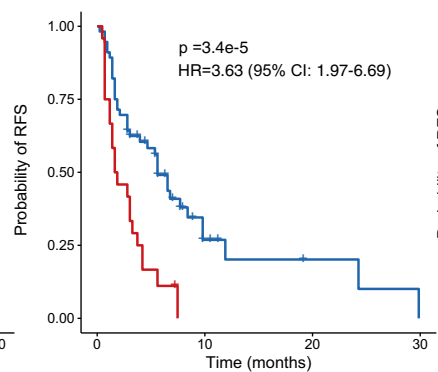

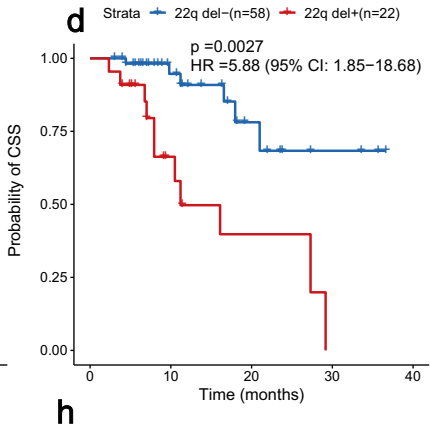

Strata $+22 q$ del- $(n=58)+22 q$ del $+(n=22)$

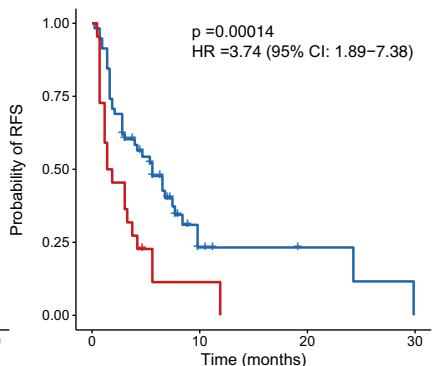

Fig. 5 Genomic features in relation to chordoma-specific survival (CSS) and recurrence-free survival (RFS). CSS (a-d) and RFS (e-h) of chordoma patients stratified by PBRM1 status, 9p21.3 deletion, 9q21.11 deletion, and 22q deletion. $p$ values, hazard ratios (HRs), and 95\% confidence intervals (Cls) were obtained from Cox proportional hazards models with the adjustment of age, sex, presurgery and post-surgery radiation therapy. "+" indicates subjects carrying the genomic alteration. See also Supplementary Table 6.

features and CSS/RFS did not change significantly with the additional adjustment of tumor KI67 status, gross resection rate, TMB, and SCNA group (Supplementary Table 6C). Associations for PBRM1 alterations and 22q deletion for both CSS and RFS remain significant when we restricted the analyses to the 53 patients who were not previously diagnosed with or treated for chordoma and who did not have presurgery RT (Supplementary Table 6C).

Paired primary and recurrence/metastasis comparison. We sequenced the genome of 11 paired primary and local recurrent tumor samples with the same sequencing depth. On average, recurrence samples showed increased number of SNVs $(30.1 \%$ higher), indels (43.1\% higher), genomic regions covered by SCNAs (2.1\% higher), and SVs (43.5\% higher), compared with the matched primary tumor samples. The time to the first recurrence (TTFR) ranged from 3 to 36 months with a median of 8 months. As expected, TTFR was positively associated with the number of SNVs (Pearson correlation $(r)=0.47, p=0.14$ ) and indels $(r=0.64, p=0.035)$ that were specific to recurrence samples (acquired during tumor progression). However, recurrent events, especially those involving known cancer driver genes, that were specific to either primary or recurrence samples were rarely observed in more than one patient.

Overall, the paired primary and recurrent tumor samples showed high numbers and proportions of shared SCNAs and, to a lesser extent, SNVs, indels (Fig. 6a, d), suggesting that SCNAs are fundamental to the initiation of chordoma. Mutational signatures and SV events also showed similar patterns between paired primary and recurrence samples for most patients (Fig. 6b, c, e).

Independently from the primary analysis of 80 patients, we analyzed a patient with metastatic chordoma and compared genomic profiles among the chordoma recurrence (R), LM, and TM samples. The total number of mutations was 1564, 1595, and 1552, for R, LM, and TM samples, respectively. Mutations in known cancer driver genes were not found in any of these samples, therefore, the mutation evolution analysis was not informative. Focal deletion of the $C D K N 2 A$ region, which was seen in all three samples, is likely the driver event for this patient (Supplementary Fig. 9). Overall, the SV/SCNA profile and mutation signatures were very similar across the three samples (Fig. 6a-c, h). Similarly, the three samples also shared high proportions of SNVs and indels (Fig. 6f, g), suggesting a monoclonal origin. Interestingly, $A P O B E C$ signatures (SBS2 and SBS13) were only present in the TM sample, while absent in R and LM samples, which is consistent with results from a recent study reporting that $A P O B E C$ mutagenesis could generate mutations late in the evolution of metastatic disease in thoracic tumors ${ }^{27}$.

\section{Discussion}

In this genomic analysis of skull-base chordoma, we described a comprehensive genomic landscape of this rare cancer and identified several genomic features that were associated with disease progression and outcomes.

We showed that skull-base chordoma was among the cancer types that had the lowest mutation rates, similar to pediatric brain tumors and leukemias ${ }^{28}$. Driver gene analysis only detected one significantly mutated driver gene in this patient cohort, PBRM1. In addition, chromosome $3 \mathrm{p} 21$, where PBRM1 and SETD2 are located, is the region with the most prevalent chromothripsis and one of the most frequently deleted regions. Given the role of PBRM1 and SETD2 in chromatin remodeling, our data suggest that epigenetic dysregulation may play an important role in chordoma development. In line with this, previous Pan-cancer analysis found that significant focal SCNA regions without known cancer genes were enriched with genes involved in epigenetic regulation ${ }^{29}$. In our study, we identified several significant focal SCNA regions that did not contain known cancer driver genes. Future mechanistic studies should follow-up genes in these focal SCNA regions to identify additional driver genes/mechanisms.

Consistent with findings from previous studies of sacral chordoma $9,19,20,30$, we found that alterations of PBRM1 and 
a

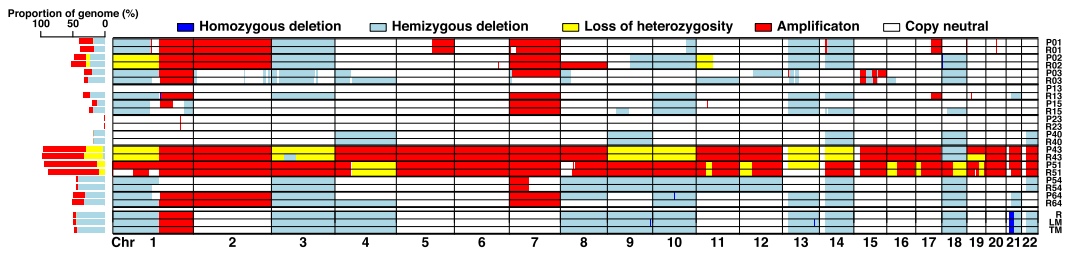

b

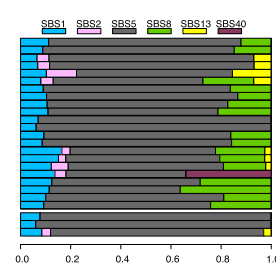

C

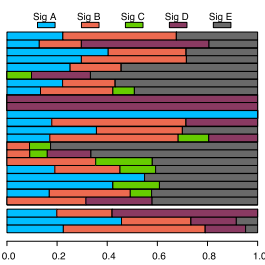

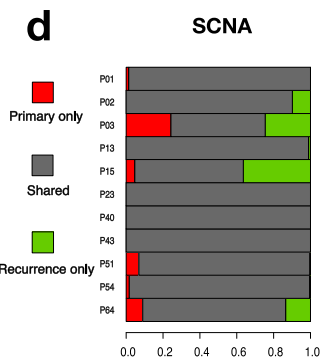

$\mathbf{f}$

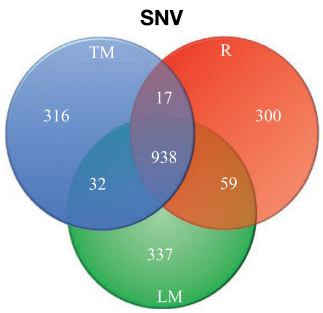

SNV

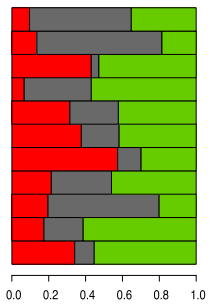

Indel

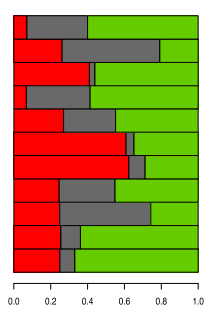

$\mathbf{e}$
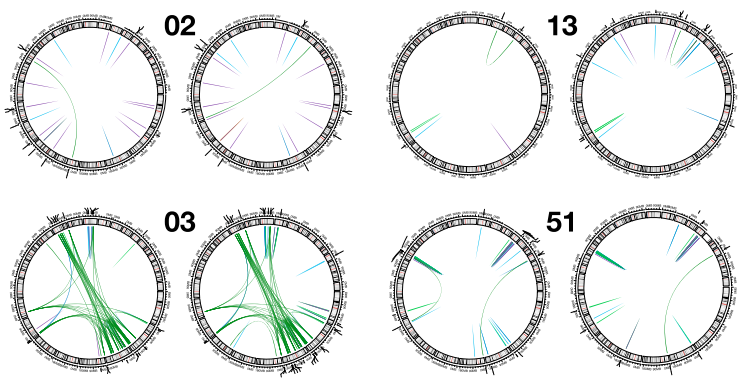

h
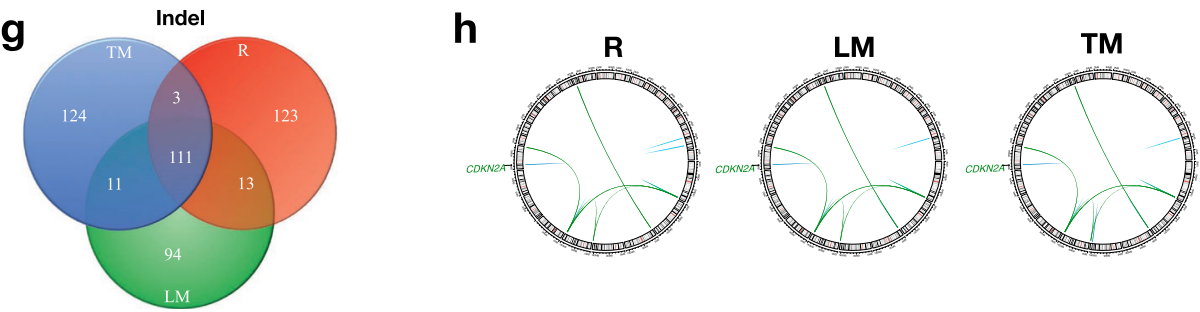

Fig. 6 Comparison of genomic alterations between paired primary tumor and recurrence/metastasis samples. Comparison of somatic copy number alterations (SCNAs, panel (a)), COSMIC single-base substitution (SBS) signatures (panel (b)), and de novo indel signatures (panel (c)) in 11 paired primary tumor $(P)$ and recurrence ( $R$ ) samples (upper) as well as three paired tumor and metastasis samples (lower). R recurrence, LM lymph node metastasis, TM thoracic metastasis. $\mathbf{d}$ Shared proportion of SCNAs, single nucleotide variations (SNVs), and indels in 11 paired primary tumor and recurrence samples. Each bar indicates the proportion of alterations in primary tumor only (red), recurrence only (green), and shared between primary and recurrence samples (gray) within each patient. e Comparison of structural variants (SVs) in four paired primary tumor and recurrence samples with total number of SVs larger than 20. Number of SNVs (f) and indels (g) shared among R, LM, and TM samples. $\mathbf{h}$ Comparison of SVs among R, LM, and TM samples. See also Supplementary Fig. 8.

$C D K N 2 A / 2 B$ locus were the most common events in chordoma. In addition, our results suggest that they occurred in a mutually exclusive manner. However, somatic duplications of TBXT and mutations in PI3K signaling genes were rare in our study, which differs from what was observed in sacral chordoma ${ }^{9}$. The discrepancies might be due to the differences in chordoma site (skull base vs. sacral), sequencing platform (WGS vs. primarily targeted), or study populations (East Asian vs. European). Higher frequencies of TBXT amplifications reported previously might be attributed to the use of higher-resolution technologies, such as high-depth sequencing and FISH, to detect low-copy gains. In addition, variations in how SCNAs are defined (arm/chromosome level vs. focal) may also cause discrepancies. In our study, although one-copy $6 \mathrm{q}$ gain was observed in nine samples (11.25\%), focal TBXT amplification was only seen in two patients. Consistent with our findings, a genome-wide SNP genotyping array analysis of skull-base chordoma also reported low frequency of focal TBXT amplification ( 1 of 18 patients $)^{20}$, suggesting that focal TBXT amplifications may be more common in sacral than skull-base chordomas. Given the low incidence as well as low mutation rates of chordoma, collaborations across different research groups such as chordoma foundation for a large-scale chordoma genomic analysis in diverse populations are needed to delineate the genomic landscape of this rare disease.
Interestingly, the comparison of mutation signature profiles between chordoma and other cancer types suggests that chordoma is most similar to kidney cancers and bone cancer. The morphological overlap between chordoma and ccRCC is known ${ }^{31}$ but the mechanism underlying this similarity remains unclear. Like chordoma, chromosome 3p loss and PBRM1 mutations are very common in sporadic ccRCCs ${ }^{32,33}$. Our findings of the similar somatic driver genes and mutation signatures in chordoma and kidney cancers suggest that there might be common etiologic factors associated with these two cancer types and identifying these factors may provide etiologic insights for chordoma.

Recently, in a WES/WGS study of 11 chordoma patients, Gröschel et al. demonstrated that SBS3, a mutational signature associated with homologous recombination deficiency (HRD) and $B R C A 1 / 2$ germline mutations, was significantly enriched in advanced chordoma samples, thus implicating the poly(ADPribose) polymerase (PARP) inhibitor as a promising therapeutic option $^{34}$. However, SBS3 was not present in any of the patients we sequenced. In addition, no pathogenic/LP germline variants in BRCA1/2 genes were found in our patients. Using HRDetect scores, which summarized six mutation features associated with HRD (SBS3, SBS8, SV signature 3, SV signature 5, HRD index, and the fractions of deletions with micro-homology) 35 , we 
identified six tumors with suggestive HRD signature, each of which was primarily driven by a single feature. None of the six tumors showed consistent HRD scores across different features, which was a characteristic of HRD observed in breast cancer. In the study by Gröschel et al., all patients had progressive disease and had previously received RT or systemic treatment prior to surgical resection, whereas few patients in our study had metastases and only a small number of patients received RT prior to surgery. Our results did not support defective HR as a common mechanism in skull-base chordoma patients.

The phylogenetic analysis was not informative due to low mutation burden and lack of driver mutations in recurrent/ metastatic tumors. However, by comparing genomes in paired primary and recurrence/metastasis samples, we found that paired samples were clonally related for all patients and the largest shared fractions between the paired samples were observed for SCNAs compared to SNVs and indels, suggesting the importance of SCNAs in tumor initiation. Nevertheless, we did find APOBEC signatures were present in distant metastatic sample but absent in recurrent tumor and lymph nodes, supporting views from recent studies that $A P O B E C$ mutations may be switched on at various stages of tumor evolution ${ }^{27}$.

Clinical outcomes in skull-base chordoma patients are highly variable and disease progression is likely determined by both surgical factors and tumor biology. Currently, there is no clear clinical guidance on patient stratification regarding treatment such as post-surgery RT. Multiple markers have been proposed, but most were based on candidate marker searches in small studies. In our study, we examined a comprehensive list of genomic alterations (including mutations, SCNAs, and SVs) and their associations with clinical outcomes with adjustment of all potential confounders. Our results suggest that PBRM1 alterations were one of the most significant prognostic factors for skullbase chordoma, which is consistent with previously reported associations between PBRM1 mutations and late stage and poor prognosis in $\mathrm{ccRCC}^{32,33}$. Notably, recent clinical data showed that PBRM1 inactivation may predict benefit from anti-PD-1 checkpoint inhibitors in ccRCC ${ }^{36}$ and Pbrm1-deficient murine melanomas were more strongly infiltrated by cytotoxic $\mathrm{T}$ cells ${ }^{37}$, suggesting that immune checkpoint inhibitors have the potential of treating chordoma patients with PBRM1 alterations.

We also found that the deletion of chromosome 22q, where SMARCB1 is located, was significantly associated with CSS and RFS. The complete loss of SMARCB1 expression is considered a hallmark of poorly differentiated chordoma, a rare and aggressive chordoma subtype ${ }^{8,26,38,39}$. Although the homozygous loss of $S M A R C B 1$ was not seen in our chordoma patients, patients with hemizygous $22 \mathrm{q}$ deletion showed reduced expression of SMARCB1 at the mRNA level. Our results suggest that partial inactivation of this gene was associated with worse patient outcomes and might be used as a prognostic marker in conventional chordoma. These findings further demonstrate the importance of SWI/SNF complex genes in chordoma initiation and progression, highlighting the need for examining SWI/SNF genes in chordoma clinics.

Consistent with previous reports, we found that 9p21 deletion was associated with worse RFS. However, complex genomic rearrangements occur on chromosome 9 , including arm-level and multiple focal deletions involving both $9 \mathrm{p}$ and $9 \mathrm{q}$. These regions showed high co-occurrence and most were associated with RFS. Surprisingly, the stronger association was observed for $9 \mathrm{q}$ deletion, especially the focal $9 q 21.11$ deletion, compared to arm-level $9 \mathrm{p}$ or $9 \mathrm{p} 21.3$ focal deletion. In particular, $C D K N 2 A / 2 B$ alterations (primarily 9p homozygous deletions) were not associated with RFS. These results suggest that the RFS association is unlikely to be entirely driven by CDKN2A inactivation. The 9q21.11 deletion peak contains hsa-mir-1299, LOC440896, PGM5P2, and FOXD4L6. Although the biological relevance remains unclear, this region warrants further investigations as a marker for recurrence in skull-base chordoma.

Remarkably, when we combined PBRM1 alterations and $22 \mathrm{q}$ deletion, the associations for both CSS and RFS became stronger, demonstrating the potential of designing a multi-marker panel in CSS prediction. Future studies with large number of patients to validate these markers in relation to disease outcomes are warranted.

Our study is still relatively small, which may have limited our ability to detect additional driver genes and de novo mutation signatures. Since RT was performed at multiple institutes/clinics, the detailed data on RT type, duration, and dosage were not available. In addition, the lack of multiple specimens from a single patient in combination with the low mutation burden has made phylogenetic analysis to study tumor evolution challenging. Despite the limitations, our study was derived from a clinically well-annotated patient population. Unlike most previous genomic studies in which advanced tumors were usually enriched, most patients in our study did not receive extensive therapies prior to surgeries and therefore the genomic profiles should not have been heavily influenced by treatment. Our analysis provided a detailed genomic landscape of skull-base chordoma, which may have important clinical implications in patient stratification and targeted treatments.

\section{Methods}

In this study, we analyzed data and biospecimens from patients who were diagnosed with skull-base chordoma and underwent endoscopic endonasal surgeries at the Neurosurgery Department of Beijing Tiantan Hospital, Capital Medical University, between October, 2010 and November, 2017. Fresh-frozen primary tumor, recurrent tumor whenever possible, and matched peripheral blood samples were collected from these patients. Clinicopathological characteristics including age, tumor histologic type, tumor volume, Ki67 status, gross resection rate, presurgery RT, post-surgery RT, recurrence, and death status were recorded using Microsoft Excel 2016 (v16.0). The chordoma diagnosis was confirmed with brachyury staining for 70 patients and was confirmed by morphology in combination with CK and EMA markers for the remaining ten patients, as illustrated in Supplementary Fig. 10 for the two patients with $I D H 1$ mutations. The study protocol was approved by the ethics committee of the Beijing Tiantan Hospital and the written informed consent was obtained for all study participants.

Biospecimen collection, quality control, and processing. A small section of frozen tumor samples was embedded in optimal cutting temperature compound, frozen with cold N-hexane, and then cut to $10-\mu \mathrm{m}$-thick frozen sections using cryostats (Leica, Germany). The frozen chordoma tissue sections were fixed in cold acetone, stained with hematoxylin and eosin, and then dehydrated through increasing concentrations of ethanol and xylene. Only tumors with $>50 \%$ tumor cells were included for DNA/RNA extraction.

DNA and RNA extractions. Genomic DNA was extracted from frozen tissue specimens and matched peripheral blood, respectively, using DNeasy blood \& tissue kit (Qiagen, CA). A total of $500 \mathrm{ng}$ DNA with high-molecular weight $(>20$ $\mathrm{Kb}$ single band) was used for the library preparation. For total RNA extraction, tissue sections were processed with TRIzol (Thermo, USA) according to the manufacture instructions. RNA was run on $1 \%$ agarose gels to check for degradation and contamination. RNA quality and quantity were assessed using the RNA Nano 6000 Assay Kit of the Bioanalyzer 2100 system (Aligent Technologies, CA, USA). RNA samples with an integrity number of over 6.8 were included for transcriptome library preparation and sequencing.

\footnotetext{
Library construction, sequencing, and data generation. WGS and RNA-Seq were carried out by the Novogene Corporation (Beijing, China). The WGS library was constructed using Truseq Nano DNA HT Sample Prep Kit (Illumina, USA) and sequenced on Illumina HiSeq X platform with the average depth of 76X for tumors and $41 \mathrm{X}$ for matched germline. After the exclusion of reads containing adapter contamination and low-quality/unrecognizable nucleotides, the clean data were mapped to the reference human genome (UCSC hg19) using the Burrows-Wheeler Aligner software (VN:0.7.8-r455, http://bio-bwa.sourceforge.net//40 to get the original mapping results stored in the BAM format. SAMtools (v1.8, http://samtools. sourceforge.net/) ${ }^{41}$, Picard(v2.18.20, http://broadinstitute.github.io/picard/), and GATK (v3.8-1-0, http://software.broadinstitute.org/gatk) ${ }^{42}$ were used to sort BAM
} 
files and to do base quality recalibration, duplicate reads removal, and local realignment to generate final BAM files for mutation calling. BAM-matcher (2016 version) ${ }^{43}$ was used to verify whether two BAM files (tumor/tumor pair or tumor/normal pair) were generated from the same patient.

Somatic variant calling. To detect somatic SNVs, five somatic callers (Strelka (v2.7.1), https://github.com/Illumina/strelka) ${ }^{44}$, Sentieon TNsnv (https://support. sentieon.com/appnotes/out_fields/) and TNhaplotyper (https://support.sentieon. com/appnotes/out_fields/), which are commercial versions of MuTect and MuTect2 ${ }^{45}$, Lofreq (V2.1.3.1, https://csb5.github.io/lofreq//) ${ }^{46}$ and MuSE (V1.0rc, https://bioinformatics.mdanderson.org/public-software/muse/ $/)^{47}$ were applied to all tumor/normal BAMs. SomaticSeq (v2.7.2, https://github.com/bioinform/ somaticseq $)^{48}$ was then applied to combine the variants called from these five callers to generate the final ensemble results. Only the variants called by two or more callers were retained. For indels, three of these callers were applied (Strelka, TNhaplotyper, and Lofreq) for indel calling and variants called by two or more callers were included in subsequent analyses. Variants were excluded if they did not pass the pipeline quality control metrics, had variant allele fraction $(\mathrm{VAF})<0.07$ in tumor, VAF $>0.02$ in normal, alternative allele read count $<3$ or total read count $<$ 8 in tumor, total read count $<6$ in normal, and if the minor allele frequency (MAF) was $>0.1 \%$ in 1000 Genomes Project ${ }^{49}$, the ESP6500 data set from University of Washington's Exome Sequencing Project (http://evs.gs.washington.edu/EVS/) or $\mathrm{ExAC}^{50}$. Manual review of variants in several genes was performed using the Integrative Genomics Viewer (v2.3.61) ${ }^{51}$.

TMB was defined as the number of somatic mutations in the coding region per megabase, including SNVs and indels. When comparing TMB in our chordoma tumors to other tumor sequenced in TCGA, we used normalized TMB, for which the distribution of variants was compiled from over 10,000 WGS samples across 33 TCGA landmark cohorts using maftools $(\mathrm{v} 1.6 .05)^{52}$.

Potential drivers. $\mathrm{dNdScv}{ }^{17}$ was used to identify driver genes, and $P B R M 1$ was the only significantly mutated gene identified in our patient cohort (FDR $<0.01)$. Since $P B R M 1$ is a SWI/SNF gene and alterations in SWI/SNF genes have been suggested to play potential driver roles in sacral chordoma, we consider genomic alterations in SWI/SNF genes, among which only alterations in PBRM1 and SETD2 (both located at chromosome $3 \mathrm{p} 21.3$ ) were identified in this patient cohort, as potential driver events. These included mutations in PBRM1 and SETD2, structural variants involving the two genes, and significant chromosome $3 \mathrm{p}$ chromothripsis regions occurring at $3 \mathrm{p} 21.3$, all of which were validated by targeted sequencing. In addition, we also considered homozygous deletion of the CDKN2A/2B locus and mutations in previously reported chordoma genes $T$ and $L Y S T^{9}$ as potential driver events. To identify driver genes or recurrently mutated genes, we focused on nonsynonymous mutations in the coding region.

Mutation validation. We selected nonsynonymous mutations in 36 genes identified by WGS, including PBRM1, SETD2, CDKN2A/2B, MAP3K4, BAG1, ITGA6, $C S D E 1$, etc., and validated these mutations using Sanger sequencing.

Mutation signature analysis. We performed mutational signature analysis for SBS, DBS, and small indels (ID) using SigProfiler (Alexandrov et al..$^{53}$ ) (v0.0.5.77, https://github.com/AlexandrovLab), as previously described in original publications ${ }^{12}$. We first performed de novo mutation signature analysis. For each extracted mutation signature, we identified a set of COSMIC signatures whose linear combinations best approximated the given de novo mutation signature. Cosine similarity index was calculated between the de novo mutation signature and the linear combination of COSMIC signatures to evaluate whether the de novo signature was novel. We then used SigProfiler to evaluate the contribution of each COSMIC signature for each tumor sample.

PyClone (v0.13.1, http://compbio.bccrc.ca/software/pyclone) ${ }^{14}$ was used to classify SNVs into clonal and subclonal SNVs, accounting for CN and purity estimated by FACETS (v0.5.6, https://github.com/mskcc/facets) ${ }^{18}$. We then performed mutation signature analysis separately for clonal and subclonal mutations to investigate mutation signatures associated with tumor initiation and progression.

Somatic copy number alteration (SCNA) analysis. Allele-specific SCNA analysis was performed using FACETS (version 0.5.6, https://github.com/mskcc/facets) ${ }^{18}$. An arm-level SCNA was defined if the SCNA event covered $90 \%$ of the p or q arm of each chromosome. GISTIC $2.0^{21}$ was used to detect significantly mutated regions. In addition, FACETS also estimated clonal status of each SCNA event based on the distribution of VAF. In our data set, $89.5 \%$ of SCNA events were estimated to be clonal. We performed GISTIC analysis separately on clonal SCNAs and subclonal SCNAs, but we did not identify additional significantly mutated regions that were specific to clonal or subclonal SCNAs.

We also used FACETS to estimate purity for samples with sufficiently informative SCNA events. For samples without informative SCNA events, we estimated purity based on SNVs. Briefly, we identified SNVs located in copy neutral regions based on FACETS and assumed the number of the mutant allele followed a mixture of binomial distributions $x_{i} \sim \sum_{k=1}^{K} \pi_{i} \operatorname{Binom}\left(x_{i} ; N_{i}, \theta_{i}\right)$ where $\theta_{K}<\ldots<\theta_{1} \leq 0.5$.
Parameters were estimated using the expectation-maximization algorithm, and the number of clones was determined by the Bayesian Information Criterion. The purity was estimated as $2 \theta_{1}$, because we used only SNVs in copy neutral regions. For samples with purity estimated successfully by both methods, the purity estimates were highly concordant with Pearson correlation coefficient $=80.4 \%$.

Hieratical clustering was performed using a function hclust based on SCNA profiles weighted by the length of each SCNA event. At each base pair, the distance between two tumors was defined as 0 if they had the same $\mathrm{CN}$ status (homozygous deletion, hemizygous deletion, copy neutral, amplification, loss of heterozygosity) and 1 otherwise. The number of clusters was determined by the Elbow method.

The allele-specific SCNA status inferred based on FACETS was used to determine the status of WGD. Specially, a tumor was considered to have undergone WGD if more than $50 \%$ of the autosomal genome had an MCN (CN of the major allele, the more frequent allele in a given segment) $\geq 2^{22}$.

Structural variant (SV) analysis. We used the Meerkat (v0.189, http://compbio. med.harvard.edu/Meerkat/) algorithm ${ }^{23}$ to call somatic SVs and estimate the corresponding genomic positions of breakpoints from recalibrated BAM files in the primary analysis. We used parameters adapted to the sequencing depth for both tumor and normal tissue samples and the library insert size. To compare results, we also called SVs with our newly developed pipeline (MoCCA-SV, v0.2, https:// github.com/NCI-CGR/MoCCA-SV) ${ }^{54}$ that integrates results from four callers, Svaba (v1.1.0) ${ }^{55}$, Breakdancer (v1.4.5) ${ }^{56}$, Delly (v0.8.1) ${ }^{57}$, and Manta (v1.4.0) $)^{58}$ The criteria for comparison across callers were $70 \%$ reciprocal overlap and $50 \mathrm{bp}$ window for intrachromosomal breakends. We focused on events called by all four callers or $\geq 3$ callers for SV candidates already found in one sample by Meerkat.

Chromothripsis analysis. We used ShatterSeek (https://github.com/parklab/ ShatterSeek) to identify chromothripsis events ${ }^{24}$. Permutation tests were performed to identify such rearrangement clusters followed by manual curation for all cases. Only regions with high interactions were included. Regions affected by chromothripsis were characterized by clusters of breakpoints belonging to SVs that are interleaved. Thus, high-confidence chromothripsis events were identified if a region satisfied one of the following sets of statistical criteria: (1) $\geq 6$ interleaved intrachromosomal SVs, seven adjacent segments oscillating between two $\mathrm{CN}$ states, the fragment joins test $p<0.05$, and either the chromosomal enrichment or the exponential distribution of breakpoints test $p<0.05$; and (2) at least three interleaved intrachromosomal SVs and four or more interchromosomal SVs, seven adjacent segments oscillating between two $\mathrm{CN}$ states and the fragment joins test $p<0.05$.

Pathogenicity scoring of germline rare variants. Three variant callers, GATK (v3.8-1-0) HaplotyperCaller, UnifiedGenotyper, and FreeBayes (v1.2.0) ${ }^{59}$ were used to call germline variants. The GATK LeftAlignAndTrimVariants module was also applied for the normalization of the variants obtained from the three callers. The majority-voting ensemble approach with bcbio (https://github.com/bcbio/bcbio. variation.recall) was applied to combine normalized variants from three callers. The variants called by two or more callers were used for further analyses.

Rare variants ( $\mathrm{MAF}<0.001$ in ExAC East Asian population) passing quality control and variant filters were evaluated for pathogenicity. A step-wise pipeline was constructed to evaluate each rare variant in the genes of interest, as previously described (Mirabello, under revision). Briefly, variants were classified as

"Pathogenic" (P), "Likely Pathogenic," "Variant of Uncertain Significance" (VUS), "Likely Benign," or "Benign" based on ClinVar (26), InterVar version 2.1.2 (default settings ${ }^{60}$, impact (frameshift indels, stop gain/loss, or known splice sites), HGMD $(2018.1)^{61}$, and manual review of the published literature. All P and LP designated variants, the high impact and HGMD DM variants were manually reviewed for a final designation of P, LP, or VUS_D.

Germline duplication of TBXT. PennCNV-seq software (v1.0.4, https://github. com/WGLab/PennCNV-Seq $)^{62}$ was performed to call germline copy number variations (CNVs) on 80 normal samples. Using BAM files and the reference genome (FASTA file), sequence counts and B allele frequencies (BAF) were generated. SAMtools was used to calculate the coverage (with mpileup) and $\log \mathrm{R}$ ratios (LRR). Population frequency of the $B$ allele for each marker was generated. Hidden Markov model in the package was used to detect potential germline CNVs and generate CNV calls. Adjacent CNV calls were merged. We removed CNV calls from samples with LRR_SD $\geq 0.35$, calls from centromeric regions, and calls with number of SNPs $<10$ or length $<1000 \mathrm{bp}$. CNV calls from region chr6:160,000,000 to $170,000,000$, where the TBXT gene is located, were extracted. Plots for calls with this region were generated and reviewed. The presence of TBXT duplication was confirmed using an independent analysis, the Canvas (v1.31) CNV caller developed by Illumina ${ }^{63}$. Briefly, Canvas scans for genomic regions with statistically significant different number of short read alignments, with the baseline estimated assuming most of the genome is diploid and reads are distributed randomly across the genome. Regions with fewer than the expected number of alignments are classified as losses. Regions with more than the expected number of alignments are classified as gains. Germline duplications covering the $T B X T$ gene were visually verified by plotting the LRR and the BAF in the genomic region. 
RNA sequencing. A total amount of $3 \mu \mathrm{g}$ RNA per sample was used as input material for RNA sample preparations. First, we removed ribosomal RNA using the Epicentre Ribo-zeroTM rRNA Removal Kit (Epicentre, USA). Subsequently, sequencing libraries were generated using the rRNA-depleted RNA and NEBNext UltraTM Directional RNA Library Prep Kit from Illumina (NEB, USA) following manufacturer's recommendations. After adapter ligation and library amplification, the library fragments were purified with AMPure XP system (Beckman Coulter, Beverly, USA) in order to select fragments of preferentially $150-200 \mathrm{bp}$ in length. The strand marked with dUTP was not amplified, allowing strand-specific sequencing. Finally, products were purified (AMPure XP system) and library quality was assessed on the Agilent Bioanalyzer 2100 system. After cluster generation, the libraries were sequenced on an Illumina Hiseq platform and $150 \mathrm{bp}$ paired-end reads were generated. Raw data of fastq format were processed and clean data were generated by removing reads containing adapters or ploy-N and low-quality reads. Gene expression was quantified as TPM (transcript per million) using RSEM (https://github.com/ deweylab/RSEM) ${ }^{64}$, and $\log _{2}$ TPM was used for statistical analyses.

Statistical analyses. The Wilcoxon rank test was used to compare mean differences in genomic alterations across different groups of patients stratified by treatment, clinical or genomic features. Multivariate regression analysis was used to assess the associations between multiple genomic features and patient characteristics, with the adjustment of age at diagnosis, sex, presurgery and post-surgery RT. The Kaplan-Meier method was used to assess RFS and CSS among patients, stratified by the different genomic events. Multivariate Cox proportional hazards model was also used to test the differences in survival outcomes across the studied genomic features with the adjustment of age at diagnosis, sex, presurgery and postsurgery RT. All statistical tests in the present study were two sided and performed using SAS version 9.4 (SAS Institute, Cary, NC, USA) or R version 3.6.1 (R Foundation for Statistical Computing, Vienna, Austria).

Reporting summary. Further information on research design is available in the Nature Research Reporting Summary linked to this article.

\section{Data availability}

The WGS and RNA-Seq data generated in this study have been deposited in the dbGaP database under Accession Code phs002301.v1.p1. The remaining data are available within the article, Supplementary information, or available from the authors upon request.

Received: 6 April 2020; Accepted: 6 January 2021; Published online: 03 February 2021

\section{References}

1. Salisbury, J. R., Deverell, M. H., Cookson, M. J. \& Whimster, W. F. Threedimensional reconstruction of human embryonic notochords: clue to the pathogenesis of chordoma. J. Pathol. 171, 59-62 (1993).

2. Boriani, S. et al. Chordoma of the mobile spine: fifty years of experience. Spine 31, 493-503 (2006)

3. Zuckerman, S. L., Bilsky, M. H. \& Laufer, I. Chordomas of the skull base, mobile spine, and sacrum: an epidemiologic investigation of presentation, treatment, and survival. World Neurosurg. 113, e618-e627 (2018).

4. Yang, X. H. R. et al. T (brachyury) gene duplication confers major susceptibility to familial chordoma. Nat. Genet. 41, 1176-1178 (2009).

5. Pillay, N. et al. A common single-nucleotide variant in $\mathrm{T}$ is strongly associated with chordoma. Nat. Genet 44, 1185-1187 (2012).

6. Kelley, M. J. et al. Characterization of T gene sequence variants and germline duplications in familial and sporadic chordoma. Hum. Genet. 133, 1289-1297 (2014).

7. Jones, P. S. et al. Outcomes and patterns of care in adult skull base chordomas from the Surveillance, Epidemiology, and End Results (SEER) database. J. Clin. Neurosci. 21, 1490-1496 (2014).

8. Frezza, A. M., Botta, L., Trama, A., Dei Tos, A. P. \& Stacchiotti, S. Chordoma: update on disease, epidemiology, biology and medical therapies. Curr. Opin. Oncol. 31, 114-120 (2019)

9. Tarpey, P. S. et al. The driver landscape of sporadic chordoma. Nat. Commun. 8, 890 (2017)

10. Rahman, N. Realizing the promise of cancer predisposition genes. Nature 505, 302-308 (2014).

11. $\mathrm{Wu}, \mathrm{Z}$. et al. The brachyury Gly177Asp SNP is not associated with a risk of skull base chordoma in the Chinese population. Int. J. Mol. Sci. 14, 21258-21265 (2013).

12. Alexandrov, L. B. et al. The repertoire of mutational signatures in human cancer. bioRxiv 322859 https://www.biorxiv.org/content/10.1101/322859v2 (2019).
13. Jager, M. et al. Deficiency of nucleotide excision repair is associated with mutational signature observed in cancer. Genome Res. 29, 1067-1077 (2019).

14. Roth, A. et al. PyClone: statistical inference of clonal population structure in cancer. Nat. Methods 11, 396-398 (2014)

15. Goncearenco, A. et al. Exploring background mutational processes to decipher cancer genetic heterogeneity. Nucleic Acids Res. 45, W514-W522 (2017).

16. Bailey, M. H. et al. Comprehensive characterization of cancer driver genes and mutations. Cell 174, 1034-1035 (2018).

17. Martincorena, I. et al. Universal patterns of selection in cancer and somatic tissues. Cell 171, 1029-1041 (2017).

18. Shen, R. \& Seshan, V. E. FACETS: allele-specific copy number and clonal heterogeneity analysis tool for high-throughput DNA sequencing. Nucleic Acids Res. 44, e131 (2016).

19. Le, L. P. et al. Recurrent chromosomal copy number alterations in sporadic chordomas. PLOS ONE 6, el8846 (2011).

20. Diaz, R. J. et al. High-resolution whole-genome analysis of skull base chordomas implicates FHIT loss in chordoma pathogenesis. Neoplasia 14, 788-798 (2012).

21. Mermel, C. H. et al. GISTIC2.0 facilitates sensitive and confident localization of the targets of focal somatic copy-number alteration in human cancers. Genome Biol. 12, R41 (2011).

22. Bielski, C. M. et al. Genome doubling shapes the evolution and prognosis of advanced cancers. Nat. Genet. 50, 1189-1195 (2018).

23. Yang, L. et al. Diverse mechanisms of somatic structural variations in human cancer genomes. Cell 153, 919-929 (2013).

24. Korbel, J. O. \& Campbell, P. J. Criteria for inference of chromothripsis in cancer genomes. Cell 152, 1226-1236 (2013).

25. The ICGC/TCGA Pan-Cancer Analysis of Whole Genomes Consortium. Pancancer analysis of whole genomes. Nature 578, 82-93 (2020).

26. Singh, A. et al. A nonchordomatous-looking chordoma: when INI-1 and radiology came to the rescue!!! J. Pediatr. Hematol. Oncol. 42, 218-219 (2020).

27. Roper, N. et al. APOBEC mutagenesis and copy-number alterations are drivers of proteogenomic tumor evolution and heterogeneity in metastatic thoracic tumors. Cell Rep. 26, 2651-2666 (2019).

28. Martincorena, I. \& Campbell, P. J. Somatic mutation in cancer and normal cells. Science 349, 1483-1489 (2015).

29. Zack, T. I. et al. Pan-cancer patterns of somatic copy number alteration. Nat. Genet. 45, 1134-1140 (2013).

30. Wang, L. et al. Genomic aberrations frequently alter chromatin regulatory genes in chordoma. Genes Chromosomes Cancer 55, 591-600 (2016).

31. Clayton, E. F., Ziober, A., Yao, Y. \& Bing, Z. Malignant tumors with clear cell morphology: a comparative immunohistochemical study with renal cell carcinoma antibody, Pax8, steroidogenic factor 1, and brachyury. Ann. Diagn. Pathol. 17, 192-197 (2013).

32. da Costa, W. H. et al. Polybromo-1 (PBRM1), a SWI/SNF complex subunit is a prognostic marker in clear cell renal cell carcinoma. BJU Int. 113, E157-E163 (2014)

33. Pawlowski, R. et al. Loss of PBRM1 expression is associated with renal cell carcinoma progression. Int J. Cancer 132, E11-E17 (2013).

34. Groschel, S. et al. Defective homologous recombination DNA repair as therapeutic target in advanced chordoma. Nat. Commun. 10, 1635 (2019).

35. Zhao, E. Y. et al. Homologous recombination deficiency and platinum-based therapy outcomes in advanced breast cancer. Clin. Cancer Res. 23, 7521-7530 (2017).

36. Miao, D. et al. Genomic correlates of response to immune checkpoint therapies in clear cell renal cell carcinoma. Science 359, 801-806 (2018).

37. Pan, D. et al. A major chromatin regulator determines resistance of tumor cells to T cell-mediated killing. Science 359, 770-775 (2018).

38. Mobley, B. C. et al. Loss of SMARCB1/INI1 expression in poorly differentiated chordomas. Acta Neuropathol. 120, 745-753 (2010).

39. Pawel, B. R. SMARCB1-deficient Tumors of Childhood: A Practical Guide. Pediatr. Dev. Pathol. 21, 6-28 (2018).

40. Li, H. \& Durbin, R. Fast and accurate short read alignment with BurrowsWheeler transform. Bioinformatics 25, 1754-1760 (2009).

41. Li, H. et al. The sequence alignment/Map format and SAMtools. Bioinformatics 25, 2078-2079 (2009).

42. McKenna, A. et al. The Genome Analysis Toolkit: a MapReduce framework for analyzing next-generation DNA sequencing data. Genome Res. 20, 1297-1303 (2010)

43. Wang, P. P., Parker, W. T., Branford, S. \& Schreiber, A. W. BAM-matcher: a tool for rapid NGS sample matching. Bioinformatics 32, 2699-2701 (2016).

44. Saunders, C. T. et al. Strelka: accurate somatic small-variant calling from sequenced tumor-normal sample pairs. Bioinformatics 28, 1811-1817 (2012)

45. Cibulskis, K. et al. Sensitive detection of somatic point mutations in impure and heterogeneous cancer samples. Nat. Biotechnol. 31, 213-219 (2013).

46. Wilm, A. et al. LoFreq: a sequence-quality aware, ultra-sensitive variant caller for uncovering cell-population heterogeneity from high-throughput sequencing datasets. Nucleic Acids Res. 40, 11189-11201 (2012). 
47. Fan, Y. et al. MuSE: accounting for tumor heterogeneity using a samplespecific error model improves sensitivity and specificity in mutation calling from sequencing data. Genome Biol. 17, 178 (2016).

48. Fang, L. T. et al. An ensemble approach to accurately detect somatic mutations using SomaticSeq. Genome Biol. 16, 197 (2015).

49. The 1000 Genomes Project Consortium. A map of human genome variation from population-scale sequencing. Nature 467, 1061-1073 (2010).

50. Lek, M. et al. Analysis of protein-coding genetic variation in 60,706 humans. Nature 536, 285-291 (2016).

51. Thorvaldsdottir, H., Robinson, J. T. \& Mesirov, J. P. Integrative Genomics Viewer (IGV): high-performance genomics data visualization and exploration. Brief. Bioinform. 14, 178-192 (2013).

52. Mayakonda, A., Lin, D. C., Assenov, Y., Plass, C. \& Koeffler, H. P. Maftools: efficient and comprehensive analysis of somatic variants in cancer. Genome Res. 28, 1747-1756 (2018).

53. Alexandrov, L. B. et al. Signatures of mutational processes in human cancer. Nature 500, 415-421 (2013).

54. Ballew, B. J., Yeager, M., Hicks, B. \& Zhu, B. MoCCA-SV: A Flexible Ensemble Framework for Structural Variant Analysis. Poster session presented at: 19th Annual General Meeting of Advances in Genome Biology and Technology (AGBT); 2019 Feb 27 - Mar 3, Marco Island FL.

55. Wala, J. A. et al. SvABA: genome-wide detection of structural variants and indels by local assembly. Genome Res. 28, 581-591 (2018).

56. Chen, K. et al. BreakDancer: an algorithm for high-resolution mapping of genomic structural variation. Nat. Methods 6, 677-681 (2009).

57. Rausch, T. et al. DELLY: structural variant discovery by integrated paired-end and split-read analysis. Bioinformatics 28, i333-i339 (2012).

58. Chen, X. et al. Manta: rapid detection of structural variants and indels for germline and cancer sequencing applications. Bioinformatics 32, 1220-1222 (2016).

59. Erik Garrison, G. M. Haplotype-based variant detection from short-read sequencing. arXiv 1207 https://arxiv.org/abs/1207.3907 (2012).

60. Li, Q. \& Wang, K. InterVar: clinical interpretation of genetic variants by the 2015 ACMG-AMP guidelines. Am. J. Hum. Genet. 100, 267-280 (2017).

61. Stenson, P. D. et al. The Human Gene Mutation Database: building a comprehensive mutation repository for clinical and molecular genetics, diagnostic testing and personalized genomic medicine. Hum. Genet. 133, 1-9 (2014).

62. de Araujo Lima, L. \& Wang, K. PennCNV in whole-genome sequencing data. BMC Bioinform. 18, 383 (2017).

63. Roller, E., Ivakhno, S., Lee, S., Royce, T. \& Tanner, S. Canvas: versatile and scalable detection of copy number variants. Bioinformatics 32, 2375-2377 (2016).

64. Li, B. \& Dewey, C. N. RSEM: accurate transcript quantification from RNA-Seq data with or without a reference genome. BMC Bioinform. 12, 323 (2011).

\section{Acknowledgements}

This research was supported by Beijing Municipal Science and Technology Commission (Z171100000117002), Research Special Fund for Public Welfare Industry of Health

(201402008), and the Intramural Research Program of the National Institutes of Health, National Cancer Institute, Division of Cancer Epidemiology and Genetics.

\section{Author contributions}

All authors of this research paper have directly participated in the planning, execution, or analysis of the study. Specifically, J.B., J.S., C.L., X.R.Y., and Y. Zhang contributed to the study conception and design. J.B., C.L., S.W., Y. Zhai, M.L., S.Z., Q.L., P.Z., S.G., and Y. Zhang contributed to patient recruitment, data and specimen collection, tissue processing, and experimental assays. J.D. and J.W. helped with the pathologic review. T.Z., B.Z.(bioinformatician), L.S., D.W., M.W., W.Z., and B.J.B. performed bioinformatics analyses. J.S., X.H., H.K., H.H.W., and B.Z. (biostatistician) conducted all the statistical analyses. B.H. and Y. Zhang provided administrative support. L.M. helped with germline rare variant classification. D.M.P. and A.M.G. provided expertise in genetic epidemiology of chordoma. J.B., J.S., and X.R.Y. were responsible for data interpretation and drafted the paper. Y. Zhang secured the funding for the sequencing analysis. X.R.Y. provided supervision in all components of the study from study design to data interpretation and critical revision of the paper.

\section{Competing interests}

The authors declare no competing interests.

\section{Additional information}

Supplementary information The online version contains supplementary material available at https://doi.org/10.1038/s41467-021-21026-5.

Correspondence and requests for materials should be addressed to Y.Z

Peer review information Nature Communications thanks Peter Campbell and the other, anonymous, reviewer(s) for their contribution to the peer review of this work. Peer reviewer reports are available.

Reprints and permission information is available at http://www.nature.com/reprints

Publisher's note Springer Nature remains neutral with regard to jurisdictional claims in published maps and institutional affiliations.

pen Access This article is licensed under a Creative Commons Attribution 4.0 International License, which permits use, sharing, adaptation, distribution and reproduction in any medium or format, as long as you give appropriate credit to the original author(s) and the source, provide a link to the Creative Commons license, and indicate if changes were made. The images or other third party material in this article are included in the article's Creative Commons license, unless indicated otherwise in a credit line to the material. If material is not included in the article's Creative Commons license and your intended use is not permitted by statutory regulation or exceeds the permitted use, you will need to obtain permission directly from the copyright holder. To view a copy of this license, visit http://creativecommons.org/ licenses/by/4.0/.

(C) The Author(s) 2021 